\title{
Finite Element Simulation Analysis for Stress States of Hot Dip Galvanized Fasteners
}

\author{
Rui LI ${ }^{1, a}$, Miao QIAN ${ }^{1, b^{*}}$, Hong-Yun YU ${ }^{1, c}$, Guo-Yong LI ${ }^{1, d}$, Min-Bo YU ${ }^{1, e}$, \\ Zhi-Hua ZHU ${ }^{1, f}$, Zhou-Xuan $\mathrm{LI}^{1, \mathrm{~g}}$ \\ ${ }^{1}$ National Electrical Equipment Safety Performance Quality Supervision and Inspection Center, \\ SGCC Electrical Equipment Safety Performance Testing Technology Lab .NO.217, Taizhou Road, \\ Hangzhou City, Zhjiang Province, China \\ alirui_51217461@163.com, b153634553@qq.com, c13958120182@163.com,

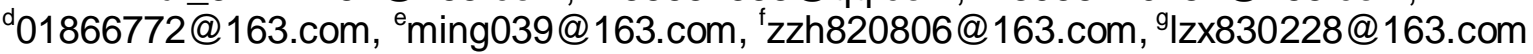

${ }^{*}$ Corresponding author

Keywords: Hot Dip Galvanizing, Fasteners, Stress State, Finite Element Analysis.

\begin{abstract}
This paper establishes models for all the components of hot dip galvanized fasteners under working conditions, and also carries out assembly modeling based on their assembly relationships. Subsequently, these operations including finite element mesh generation, restriction of boundary conditions, definition of calculation rules and solution-seeking are carried out on the assembled model. Finally, the calculated results are analyzed. According to the final analysis, the failure of hot dip galvanized fasteners is primarily the breakage caused by equivalent stress es, and the dangerous stress point is located at the root of external threads. The maximum stress on nut is larger than that on bolt.
\end{abstract}

\section{Introduction}

Hot dip galvanized fasteners are extensively used in the mechanical and electrical industries because of their satisfactory anti-corrosion performance and low prices[1 2]. However, as a bolted connection, the tightening stress state of hot dip fasteners is very complicated. It is difficult to make researches with simplified models in analyzing its failure or dangerous stress points, while finite element proves to be an effective method to simulate the stress and its changes as well as the failure behaviors. This paper investigates the stress changes, dangerous stress points and failure patterns of hot dip galvanized fasteners under working conditions by using 3D modeling software and finite element analysis software. Refer to Fig.1 for the calculation methods.

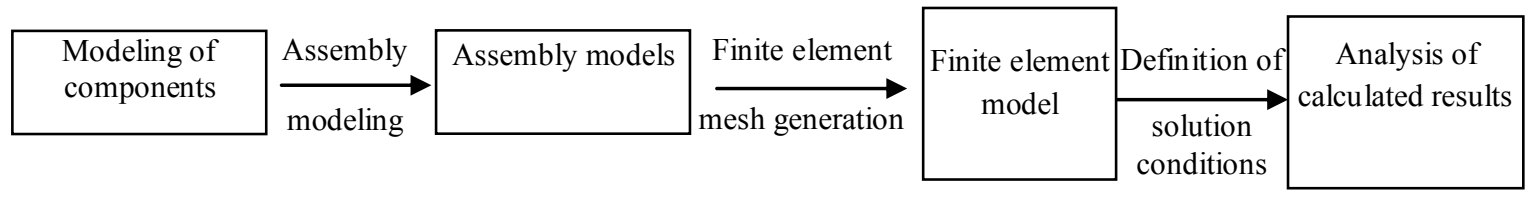

Fig. 1 Finite Element Model Analysis and Calculation Method

\section{Geometric Modeling}

In this paper, common M20-6.8 hot dip galvanized fasteners are chosen for modeling analysis. At first, 3D modeling software is used to design and establish models for major components of fasteners. The designed components mainly include a bolt, a nut, a sleeve and a part to be connected. 3D models are shown from Fig.2 (a) through (d). Next, assembly modeling is made for the designed components based on their assembly relationships. The three-dimensional diagram of assembled model is as shown in Fig.3. 


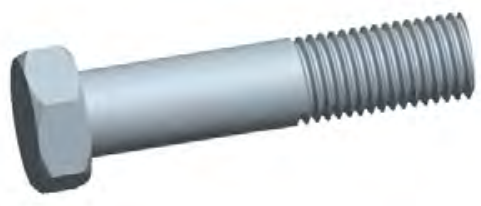

(a)

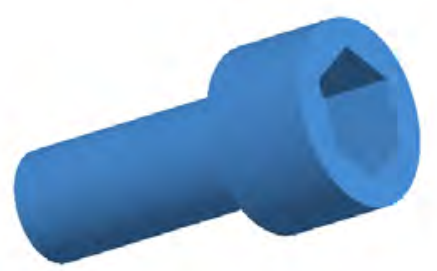

(c)

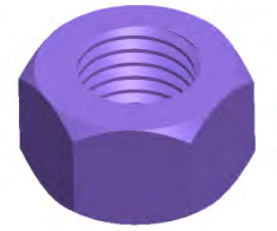

(b)

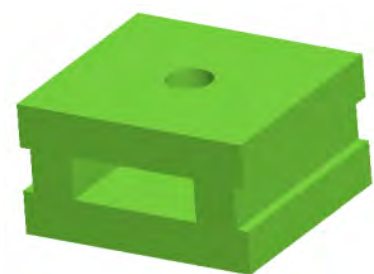

(d)

(a) Bolt; (b) Nut; (c) Sleeve; (d) the Part to be Connected

Fig. 2 Component Modeling Diagrams

(1) Bolt. The bolt model is as shown in Fig.2(a), formed by rotating, stretching, chamfering and spiral cutting.

(2) Nut. The nut model is as shown in Fig.2(b), formed by stretching, chamfering and spiral cutting.

(3) Sleeve. The sleeve model is as shown in Fig.2(c), formed by rotating and stretching.

(4) The part to be connected. Its model is as shown in Fig.2(d), formed by stretching and rotating.

(5) Assembly of models. Assembly is made according to the relationship of various components, as shown in Fig.3.

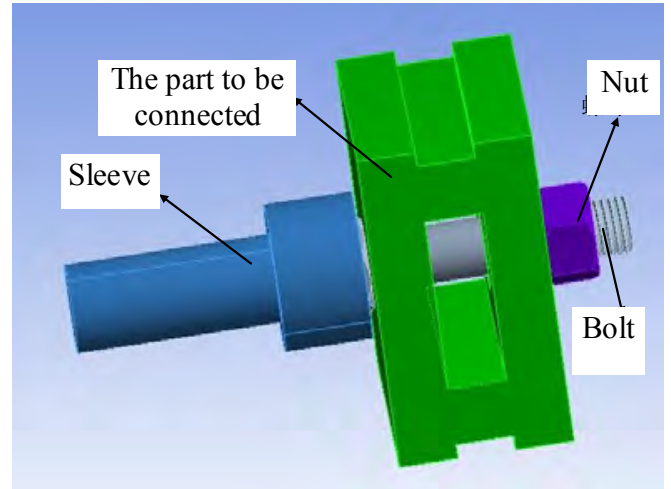

Fig. 3 Three-dimensional Assembly Model

\section{Modeling for Finite Element Analysis}

After the individual component models and assembly model for the bolt stress system are established, finite element analysis documents are to be developed, and 3D model is introduced to define the positions of components and stress constraints based on the contact of stress models and the stress conditions. In addition, mesh generation is carried out on the finite element model, and material properties, calculation and solution rules are defined for simulation analysis.

\section{Stress Constraints}

The specific performance parameters of M20-6.8 hot dip galvanized fasteners are given in Table 1. The working conditions are mimicked, and the stress analysis of fasteners during tightening is as shown in Fig.4, and the nut is suffering the tightening torques, and the stresses and torques from the part to be connected. The part to be connected is suffering the stresses and torques from the nut. Both sides of the part to be connected are fixed, and thus the stress is applied according to the 
original value.

Tab. 1 Performance Parameters of M20-6.8 Hot Dip Galvanized Fasteners[3]

\begin{tabular}{|c|c|c|c|}
\hline \multicolumn{2}{|c|}{ Given Conditions } & \multicolumn{2}{c|}{ Assumptions } \\
\hline Grade & M20-6.8 & Friction coefficient between threads $\mu_{\mathrm{s}}$ & 0.40 \\
\hline Nominal tensile strength $\sigma_{\mathrm{b}}(\mathrm{MPa})$ & 600 & Friction coefficient between supporting surfaces $\mu_{\mathrm{w}}$ & 0.27 \\
\hline Nominal yield strength $\sigma_{\mathrm{s}}(\mathrm{MPa})$ & 480 & Torque coefficient $\mathrm{K}$ & 0.51 \\
\hline Nominal shear strength $\tau_{0}(\mathrm{MPa})$ & $\geq 370$ & Axial yield tightening stress N & 50307 \\
\hline
\end{tabular}

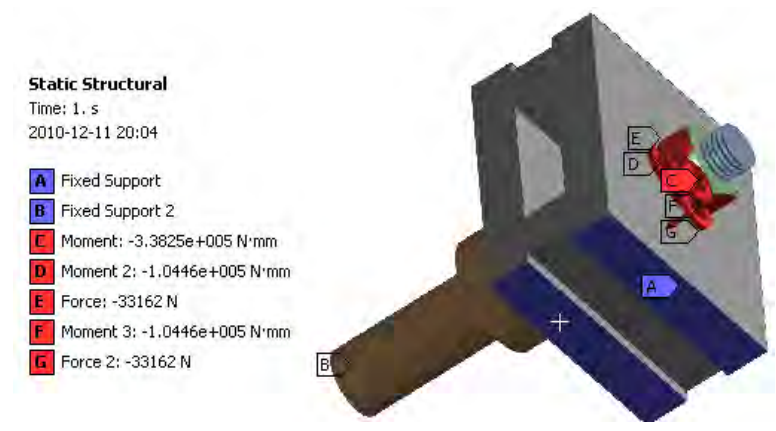

Fig. 4 Schematic Diagram for Stress Analysis of Fastening Structure

\section{Finite Element Mesh Generation}

Generally, the smaller the finite element mesh is, the more accurate the results will be. However, this requires more time for analysis. To compromise between the accuracy and analysis time, small meshes are chosen for the parts with complicated structures, while relatively large meshes for some simple components[4]. According to preliminary calculation, the deviations between analysis results are small when the mesh size is $2 \mathrm{~mm}$, indicating that the calculation accuracy is satisfactory. Therefore, the mesh size of bolts and nuts is determined to be $2 \mathrm{~mm}$, while that of sleeve and the part to be connected is $8 \mathrm{~mm}$. The mesh generation for the bolt, nut, sleeve and the part to be connected is as shown from Fig.5(a) through (d). The finite element mesh generation for the bolted connection assembly is as shown in Fig. 6.
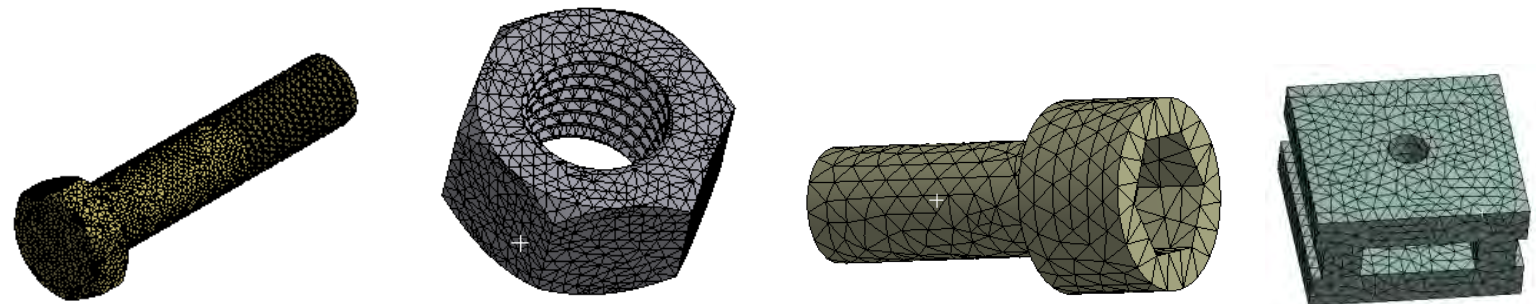

(a) Bolt Model (b) Nut Model (c) Sleeve Model (d) Model for the Part to be Connected

Fig. 5 Finite Element Mesh Generation for Models

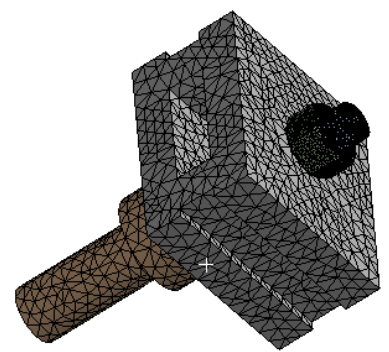

Fig. 6 Finite Element Mesh Generation for the Fastener Assembly 


\section{Stress Analysis for Bolts}

When an external moment of $338.249 \mathrm{Nm}$ is applied on a M20-6.8 bolt, the axial load may reach $33,162 \mathrm{~N}$. In this case, the axial stress, shear stress and equivalent stress on the bolt cross section are as shown from Fig. 7 through Fig. 9.

According to Fig. 7 through Fig. 9, the maximum axial stress, maximum shear stress and maximum equivalent stress of the bolt cross section all occur at the root of the threads which are engaged with the nut (for simplicity, the engaged nut is omitted in the diagram). This indicates that the root of threads is the stress center, namely, the dangerous stress point.

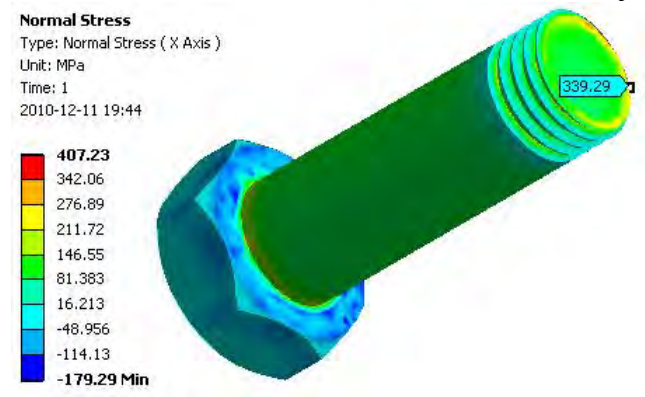

Fig. 7 Schematic Diagram for Axial Stress Distribution on Bolt Cross Section

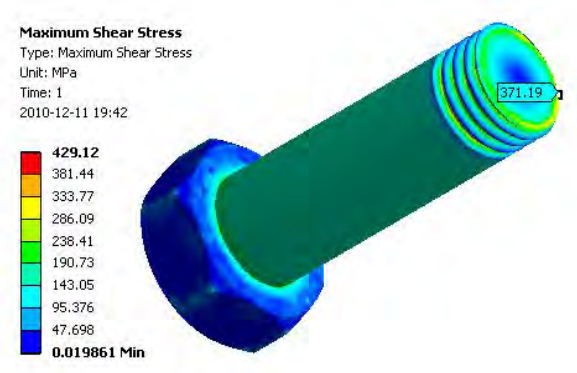

Fig. 8 Schematic Diagram for Shear Stress Distribution on Bolt Cross Section

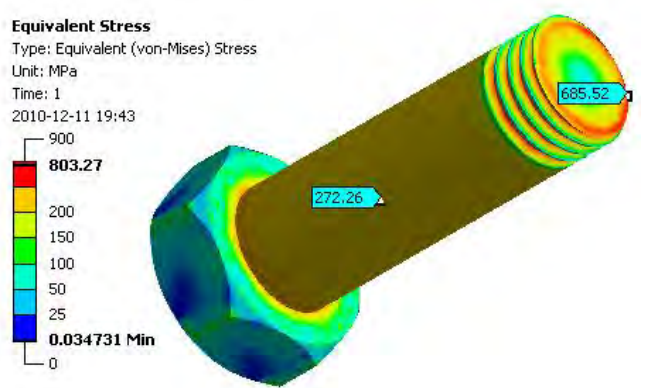

Fig. 9 Schematic Diagram for Equivalent Stress Distribution on Bolt Cross Section

In analyzing the three maximum values, we can see that the maximum axial stress $\sigma \mathrm{n}=339.29$ $\mathrm{MPa}<\sigma \mathrm{s}$, and the maximum shear stress $\tau \max =371.19 \mathrm{MPa} \approx \tau 0$, while the maximum equivalent stress $\sigma$ evmax $=685.52 \mathrm{MPa}>\sigma \mathrm{b}$. This indicates that when the maximum axial stress and the maximum shear stress on the bolt haven't yet reached the failure state or approximate the failure state, the maximum equivalent stress at the root of threads that are engaged with the nut has reached the failure state, directly causing large plastic deformation on threads. Meanwhile, partial stress has been as large as theoretical failure state, and the bolt gets failed if additional load is further applied.

\section{Stress Analysis for Nut}

When the maximum shear stress of bolt reaches the shear strength $(510 \mathrm{MPa})$, the equivalent stress and the maximum shear stress on M20-6.8 hot dip galvanized nut are as shown in Fig. 10 and Fig. 11.

According to Fig. 10, the maximum equivalent stress $\sigma_{\mathrm{ev}}^{\prime}$ on nut is $803.27 \mathrm{Mpa}$, increased by 117.75 Mpa compared with the maximum equivalent stress $\sigma_{\text {ev }}$ on bolt, 685.52 Mpa. From Fig. 11 , we can see that the maximum shear stress $\tau_{\max }^{\prime}$ on nut is $479.12 \mathrm{Mpa}$, increased by $107.93 \mathrm{Mpa}$ when compared to the maximum shear stress $\tau \max$ on bolt, 371.19 Mpa. Since the maximum equivalent stress and the maximum shear stress in stress system always appear on the nut during tightening, the strength grade of nut shall be higher than that of bolt so as to match the load and life of bolt and nut. 


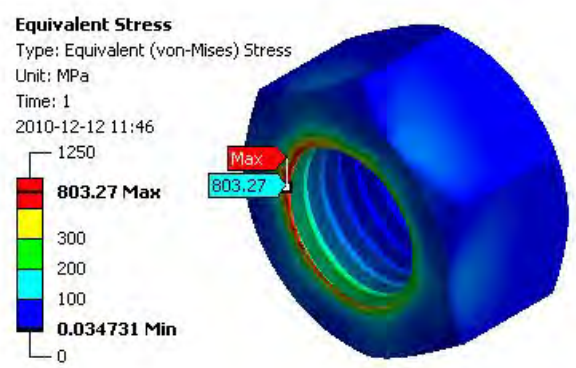

Fig.10 Equivalent Stress Distribution on Nut

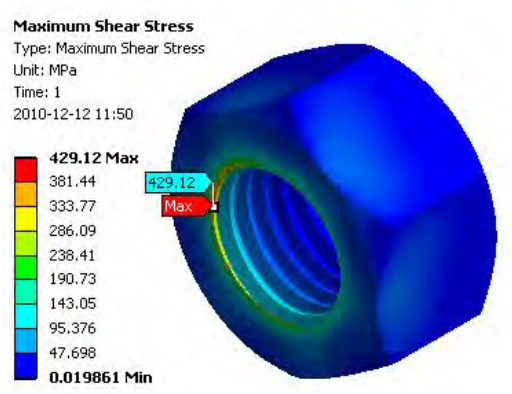

Fig. 11 Maximum Shear Stress Distribution on Nut

\section{Conclusions}

(1) Stress calculation method for hot dip galvanized fasteners is developed by using 3D modeling and finite element analysis software.

(2) According to the results of finite element simulation analysis, the bolt failure is primarily due to the breakage caused by the maximum equivalent stress, that is to say, before the axial load reaches the axial yield tightening stress, the partial maximum equivalent stress of bolt has reached the breakage strength, and thus more safety margin shall be reserved when designing the axial tightening stress.

(3) The dangerous stress point of bolt is located at the root of threads, and thus cracks at the root of threads shall be avoided or stress concentration at the root shall be avoided during the production process.

(4) The maximum stress load of hot dip galvanized fasteners lies on the nut, thus the strength grade of nut shall be higher than that of bolt.

\section{Acknowledgement}

Project Supported by Science and Technology Project of SGCC (ZDK118-2010).

\section{References}

[1]Li Zhu, Hot Dip Galvanized Steel Plate and Its Development, Angang Technology[J], 1999, 3 : $57-62$.

[2]Lixian Miao, Li Bin, Yang Tongfa, et al. Optimization of Hot Dip Galvanizing Process for Steel Parts on Power Towers, Electroplating \& Finishing[J]. 2014, 33(12): 514-516.

[3]GB/T 3098.1-2000, Mechanical Properties of Fasteners Including Bolts, Screws and Studs [S].

[4]Ping'an Du, Basic Principles of Finite Element Mesh Generation, Mechanical Design and Manufacturing [J].2000, (1): 34-36. 